\title{
Growth Performance and Post-Weaning Diarrhea in Piglets Fed a Diet Supplemented with Probiotic Complexes
}

\author{
Xuhong Lu ${ }^{1}$, Ming Zhang ${ }^{2}$, Liang Zhao ${ }^{1}$, Keshan Ge ${ }^{1}$, Zongyi Wang ${ }^{3}$, Luo Jun ${ }^{4}$, and Fazheng Ren ${ }^{1 *}$ \\ ${ }^{1}$ Beijing Advanced Innovation Center for Food Nutrition and Human Health, College of Food Science E Nutritional Engineering, China \\ Agricultural University, Beijing 100083, P.R. China \\ ${ }^{2}$ School of Food and Chemical Engineering, Beijing Technology and Business University, Beijing 100083, P.R. China \\ ${ }^{3}$ College of Food Science and Engineering, Beijing University of Agriculture, Beijing 102206, P.R. China \\ ${ }^{4}$ Swine Breeding Center of Beijing Resource Group, Beijing 102600, P.R. China
}

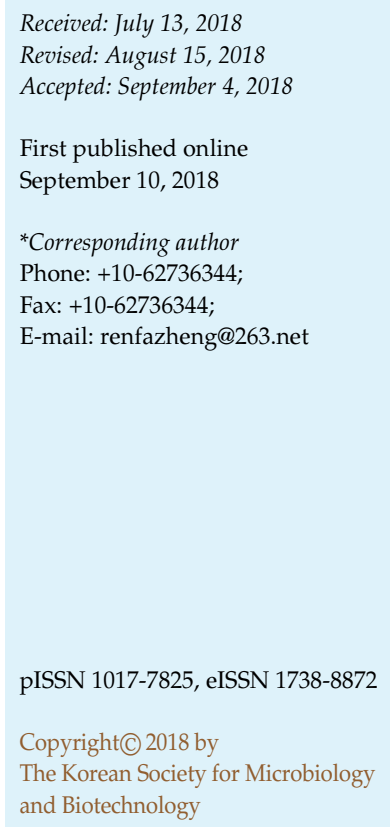

\begin{abstract}
Weaning stress can affect the growth performance and intestinal health of piglets. Dietary alternatives to antibiotics, such as dietary probiotics, especially those containing multiple microbial species, are a preventive strategy for effectively controlling post-weaning diarrhea. In this study, we investigated forty-eight crossbred piglets in three treatment groups for 21 days: the control and experimental groups were supplemented with Enterococcus faecium DSM 7134, Bacillus subtilis AS1.836 plus Saccharomyces cerevisiae ATCC 28338 (EBS) or Lactobacillus paracasei L9 CGMCC No. 9800 (EBL). On day 21, weaned piglets supplemented with two kinds of probiotic complexes showed increased growth performance and significantly reduced postweaning diarrhea $(p<0.05)$. The EBS treatment increased acetic acid and propionic acid in the feces $(p<0.05)$, and the EBL treatment increased fecal acetic acid, propionic acid, butyrate and valerate $(p<0.05)$. Moreover, the fecal microbiota of the piglets changed markedly in EBL treatment. The addition of EBS and EBL may have similar effects on the prevention of diarrhea by improving the intestinal morphology and regulating the microbiota during the weaning period.
\end{abstract}

Keywords: Probiotic complexes, piglets, post-weaning diarrhea, short-chain fatty acids, fecal microbiota

\section{Introduction}

Post-weaning diarrhea (PWD) is the most common disease in weaned piglets and is a major economic problem worldwide. PWD is mainly caused by conditioned pathogens and enterotoxins, with a high incidence of intestinal microbiota disturbances [1, 2]. Impaired gut microbiota composition after weaning can lead to slower growth, suggesting a greater permissiveness to pathogen colonization and induction of pro-inflammatory status. A recent study found a relationship between the fecal microbiota of postweaning pigs and PWD [3]. Improving the gut microbiota to avoid dysbiosis is a potential method of controlling PWD.
Dietary supplementation with antibiotics is the most effective strategy for preventing PWD. However, with increasing public concern regarding antibiotic resistance, banning antibiotics as growth promoters has been proposed in China [4]. Thus, finding acceptable alternatives to antibiotics is important for the livestock industry. As a safe and feasible replacement for antibiotics in commercial animal production, dietary probiotics are a promising preventive strategy for the control of PWD in piglets [5].

Probiotic bacteria have been shown to improve growth performance and health in weaned piglets [2]. In addition, dietary supplementation with probiotics can improve the intestinal barrier function of piglets by altering immune function [6] and intestinal microbiota [7]. These benefits are 
produced by the combined effects of a number of different probiotics [8].

Many studies have shown that different probiotic complexes have various probiotic properties and can be used to improve the health status of weaned piglets. For example, the combination of Lactobacillus casei and Enterococcus faecalis was found to significantly increase average daily gain and decrease the diarrhea rate and mortality [9]. Supplementation with a bacteria-yeast complex was shown to result in better growth performance and lower incidence of diarrhea compared with supplementation with lactic acid bacteria alone [10].

Enterococcus faecalis can improve the efficiency of feed utilization and can balance the microbial environment in weaned piglets [11]. Bacillus pumilus spore suspension can lower the incidence of diarrhea and improve growth performance after weaning [12]. Lactobacillus reuteri can also promote growth, reduce diarrhea and regulate the immune system of pigs [8]. Complexes combining these different kinds of probiotics might be more beneficial to growth performance and PWD control than individual microbial species alone during the weaning period.

The objective of the present study was to evaluate the effects of dietary probiotic complexes containing Enterococcus faecalis and Bacillus subtilis plus either Saccharomyces cerevisiae or Lactobacillus on growth performance, PWD, fecal shortchain fatty acid levels and microbiota in weaned piglets.

\section{Materials and Methods}

\section{Animal Care}

All husbandry practices and euthanasia in this experiment were performed with full consideration of animal welfare, and were approved by the Laboratory Animal Welfare and Animal Experimental Ethical Committee of China Agricultural University (CAU20170603-3).

\section{Diet and Feeding}

Forty-eight crossbred piglets (Duroc $\times$ Landrace $\times$ Large Yorkshire) weaned at $28 \mathrm{~d}$ of age, with an average initial body weight of $7.75 \pm 0.20 \mathrm{~kg}$, were randomly assigned to one of three dietary treatments (four replicates and four pigs per pen). All piglets were housed indoors on hard plastic slatted flooring. The temperature of the nursery room was initially controlled at approximately $27^{\circ} \mathrm{C}$ and was progressively decreased by $1^{\circ} \mathrm{C}$ each week. The humidity was approximately $60 \% \pm 5 \%$ and the lighting was natural. Feed and water were available throughout the 21-d feeding trial. The basal diet was formulated to meet or exceed the nutrient requirements of 7 to $11 \mathrm{~kg}$ pigs. No antibiotics were used in any diet. The ingredients and chemical composition of the basal diet are presented in Table 1.

\section{Grouping Design}

The control diet was a corn-soybean meal basal diet which included $1 \mathrm{~g}$ whey powder $/ \mathrm{kg}$ basal diet (control treatment). The probiotic complex was mixed into the diet with whey powder as a carrier. The diets with probiotic complex supplementation were

Table 1. Ingredients and nutrient content of the basal diet ${ }^{1}$.

\begin{tabular}{|c|c|c|c|}
\hline Ingredients & $\%$ & Nutrient content & $\%$ \\
\hline Extruded maize & 24.38 & Digestible energy, $\mathrm{kcal} / \mathrm{g}$ & 3.45 \\
\hline Extruded broken rice & 25.00 & Crude protein & 19.50 \\
\hline Soybean meal (46\% CP) & 22.20 & Crude fat & 7.50 \\
\hline Soybean protein concentrate $(53 \% \mathrm{CP})$ & 5.30 & Lysine & 1.54 \\
\hline Milk replacer $(42 \% \mathrm{CP})$ & 10.00 & Methionine & 0.45 \\
\hline Sweet whey $(11 \%$ CP) & 10.00 & Methionine + cystine & 0.97 \\
\hline Vitamin-mineral premix & 0.23 & Threonine & 0.96 \\
\hline Lysine $\mathrm{HCl}$ & 0.10 & Tryptophan & 0.27 \\
\hline Methionine & 0.05 & Calcium & 0.98 \\
\hline Limestone & 1.11 & Available phosphorusa & 0.49 \\
\hline Monocalcium phosphate & 1.13 & & \\
\hline Total & 100.00 & & \\
\hline
\end{tabular}

${ }^{1}$ Providing the following amounts of vitamins and minerals per kilogram on an as-fed basis: $\mathrm{Zn}$ ( $\left.\mathrm{ZnO}\right), 50 \mathrm{mg} ; \mathrm{Cu}(\mathrm{CuSO} 4), 20 \mathrm{mg} ; \mathrm{Mn}$ (MnO), $55 \mathrm{mg}$; Fe (FeSO4), 100 mg; I (KI), 1 mg; Co (CoSO4), 2 mg; Se (Na2SeO), 0.3 mg; vitamin A, 8255 IU; vitamin D3, 2000 IU; vitamin E, 40 IU; vitamin B1, 2 mg; vitamin B2, 4 mg; pantothenic acid, 15 mg; vitamin B6, 10 mg; vitamin B12, 0.05 mg; vitamin PP, 30 mg; folic acid, 2 mg; vitamin K3, 1.5 mg; biotin, 0.2 mg; choline chloride, 800 mg; and vitamin C, $100 \mathrm{mg}$. 
formulated with $1 \mathrm{~g}$ probiotic complex $/ \mathrm{kg}$ basal diet. Supplementation with two experimental probiotic complexes was evaluated. The first probiotic complex (EBS treatment) was a commercial product containing Enterococcus faecium DSM $7134\left(1 \times 10^{8} \mathrm{CFU} / \mathrm{g}\right)+$ Bacillus subtilis AS1.836 $\left(2 \times 10^{10} \mathrm{CFU} / \mathrm{g}\right)+$ Saccharomyces cerevisiae ATCC $28338\left(1 \times 10^{10} \mathrm{CFU} / \mathrm{g}\right)$. The second probiotic complex $(\mathrm{EBL}$ treatment) contained Enterococcus faecium DSM $7134\left(1 \times 10^{8} \mathrm{CFU} / \mathrm{g}\right)$ + Bacillus subtilis AS1.836 $\left(2 \times 10^{10} \mathrm{CFU} / \mathrm{g}\right)+$ Lactobacillus paracasei L9 $\left(1 \times 10^{10} \mathrm{CFU} / \mathrm{g}\right)$.

The probiotic complex supplements were both purchased from Beijing He Yiyuan Biotechnology Company. Lactobacillus paracasei L9 (CGMCC No. 9800) was isolated from human intestine [13].

\section{Growth Performance}

Feed and piglets were weighed individually at the beginning of the study and on day 21. The amount of feed was monitored; any wasted feed was also weighed and this amount was subtracted from the amount of feed added to the feeder to determine feed disappearance. The average daily gain (ADG), average daily feed intake (ADFI) and feed conversion ratio (FCR) were then calculated.

\section{Fecal Score, Moisture Content and pH Values}

Fecal consistency within each pen was visually assessed during the study by a technician who was blind to dietary treatments, according to a modification of the method described by Pierce $e t$ al. [14] as follows: hard feces $=1$; slightly soft feces in pen $=2$; soft, partially-formed feces $=3$; loose, semi-liquid feces $=4$; watery, mucoid feces $=5$. The moisture content of fecal samples was measured by vacuum freeze-drying. The $\mathrm{pH}$ of fecal samples was measured directly with a combined $\mathrm{pH}$ electrode.

\section{Sampling and Measurements}

On day 1 and day 21, fresh fecal samples were collected from each piglet. Feces were placed in a container with stabilization solution (RNAlater, a saturated ammonium sulfate solution $[\mathrm{pH}$ 5.2] containing $20 \mathrm{ml}[0.5 \mathrm{M}]$ EDTA solution and $25 \mathrm{ml}[1 \mathrm{M}]$ sodium citrate solution). Fresh fecal samples were also immediately frozen in liquid nitrogen and stored at $-80^{\circ} \mathrm{C}$.

At the end of the 21-d experiment, three piglets with similar body weight were randomly chosen from each group and euthanized. The abdomen was aseptically opened and the duodenum (about $10 \mathrm{~cm}$ distal to the pylorus) and jejunum (the middle portion of the small intestine) were sampled according to the methods described by Yang et al. [15].

\section{Determination of Short-Chain Fatty Acids in Fecal Samples}

Short-chain fatty acids (acetic acid, propionic acid and butyric acid) were quantified with gas chromatography [16]. The gas chromatography system consisted of a 7890A equipped with a flame ionization detector (Agilent, USA). The chromatographic column was HP-FFAP ( $25 \mathrm{~m}, 0.32 \mathrm{~mm}, 0.5 \mathrm{um}$ ) (Agilent, USA).

\section{DNA Extraction and High-throughput Sequencing of Fecal Samples}

Microbial DNA was extracted from 250mg fecal samples according to the methods $[6,16]$, and the V3-V4 region of the $16 \mathrm{~S}$ ribosomal RNA gene was amplified by polymerase chain reaction $\left(95^{\circ} \mathrm{C}\right.$ for $2 \mathrm{~min}$, followed by 20 cycles at $94^{\circ} \mathrm{C}$ for $30 \mathrm{sec}, 48^{\circ} \mathrm{C}$ for $30 \mathrm{sec}$, and $72^{\circ} \mathrm{C}$ for $2 \mathrm{~min}$ ) using primers $338 \mathrm{F5}^{\prime}$-(ACTCCTACG GGAGGCAGCAG)-3' and 806R 5'-(GGACTACHVGGGTWTCTA AT)-3', by thermocycler PCR system (GeneAmp 9700, ABI, USA). High-throughput sequencing of the community structure of fecal samples was performed on an Illumina MiSeq platform (Illumina, USA) according to the standard protocols by Majorbio Bio-Pharm Technology Co. Ltd. (China), while Operational Taxonomic Units (OTUs) were clustered with $97 \%$ similarity cut off using UPARSE (version $7.1 \mathrm{http}: / /$ drive5.com/uparse/). The relative abundance at the family and genus levels was compared between the two treatments with the top 10 most abundant families defined as predominant, and sorted for comparison. One-way analysis of variance (ANOVA) and other calculations were performed with the SPSS software, version 20.0. The principal coordinate analyses (PCOA) based on unweighted UniFrac distances presence discrepancy was conducted using the SAS 9.1 software to evaluate differences in the fecal microbiota between sample groups.

\section{Results}

\section{Growth Performance}

Compared with the control treatment, piglets fed a basal diet supplemented with either EBS or EBL probiotic

Table 2. Effects of probiotics on BW, ADG, ADFI and FCR of piglets on day 21 after weaning.

\begin{tabular}{|c|c|c|c|}
\hline Treatment & Control & EBS & EBL \\
\hline BW,kg & $13.8 \pm 2.19^{a}$ & $14.5 \pm 1.24^{\mathrm{b}}$ & $14.9 \pm 1.31^{b}$ \\
\hline ADG,g & $383 \pm 16.77^{a}$ & $398 \pm 18.62^{b}$ & $417 \pm 18.55^{\mathrm{b}}$ \\
\hline ADFI,g & $557 \pm 20.02^{a}$ & $538 \pm 18.62^{\mathrm{b}}$ & $520 \pm 17.98^{b}$ \\
\hline FCR,g/g & $1.45 \pm 0.08^{\mathrm{a}}$ & $1.31 \pm 0.12^{\mathrm{b}}$ & $1.27 \pm 0.13^{b}$ \\
\hline
\end{tabular}

${ }^{a b c}$ Means with different superscripts differ significantly $(p<0.05)$.

Control, basal diet supplemented with whey powder and no bacteria; EBS, basal diet supplemented with EBS complex (Enterococcus faecium DSM 7134, Bacillus subtilis AS1.836 and Saccharomyces cerevisiae ATCC 28338); EBL, basal diet supplemented with EBL complex (Enterococcus faecium DSM 7134, Bacillus subtilis AS1.836 and Lactobacillus paracasei L9). 
Table 3. Effect of probiotics on fecal score, fecal moisture content and fecal $\mathrm{pH}$ values of weaned piglets.

\begin{tabular}{lccc}
\hline \multicolumn{1}{c}{ Treatment } & Control & EBS & EBL \\
\hline Fecal score & $3.69 \pm 0.87^{\mathrm{a}}$ & $2.56 \pm 1.26^{\mathrm{b}}$ & $2.5 \pm 0.97^{\mathrm{b}}$ \\
Fecal moisture content $(\%)$ & $73.93 \pm 3.17^{\mathrm{a}}$ & $71.44 \pm 2.98^{\mathrm{b}}$ & $70.54 \pm 3.12^{\mathrm{b}}$ \\
Fecal pH & $6.93 \pm 0.76^{\mathrm{a}}$ & $6.57 \pm 0.74^{\mathrm{a}}$ & $6.32 \pm 0.59^{\mathrm{b}}$ \\
\hline
\end{tabular}

${ }^{\text {abc }}$ Means with different superscripts differ significantly $(p<0.05)$. Fecal score scale: hard feces $=1$; slightly soft feces in pen $=2$; soft, partially-formed feces $=3$; loose, semi-liquid feces $=4$; watery, mucoid feces $=5$. The moisture content of fecal samples was measured by vacuum freeze-drying.

complexes had higher body weight (BW) and ADG $(p<0.05)$ and lower ADFI and FCR $(p<0.05)$ at day 21 after weaning (Table 2). However, there were no differences in BW, ADG, ADFI or FCR between the two probiotic groups $(p>0.05)$ (Table 2). Both probiotic complexes improved the growth performance of piglets during the $21 \mathrm{~d}$ after weaning.

\section{Fecal Score, Moisture Content and $\mathrm{pH}$ Values}

Fecal scores, moisture content and $\mathrm{pH}$ values correlated with the severity of diarrhea, as summarized in Table 3. A high fecal score $(3.69 \pm 0.87)$ was observed in the control treatment. The fecal scores of weaned piglets in the EBS and EBL treatments were significantly lower than those in the control treatment $(p<0.05)$. Compared with the control treatment, both the EBS and EBL treatments had significantly lower fecal moisture content $(p<0.05)$. Feeding the probiotic EBL resulted in lower $\mathrm{pH}$ values than in the control or EBS treatments $(p<0.05)$. These findings suggest that the probiotic complexes EBS and EBL were powerful agents for controlling PWD.

\section{Short-Chain Fatty Acids}

Short-chain fatty acids (SCFAs) are produced by microbial fermentation and play an important role in digestion and absorption. The content of SCFAs in the feces of weaned piglets supplemented for 21 days was determined. Our results (Table 4) showed that levels of acetic acid and propionic acid were significantly higher in the fecal samples of weaning pigs that received EBS and EBL treatments than in controls $(p<0.05)$. The EBL treatment also significantly increased butyrate and valerate in fecal samples compared with both control and EBS treatments. The results show that supplementation with EBS or EBL probiotics had effects on the production of SCFAs in fecal samples.

\section{High-throughput Sequencing}

The effect of different probiotic treatments on the fecal microbiota of weaned piglets on day 1 and day 21 was evaluated with 16S rRNA gene amplicon sequencing (Table 5). The results of the analyses of the OTUs, Chao1 and Shannon diversity indices are presented in Table 5. Overall feces samples' coverage was about $98 \%$, indicating that the majority of microbial phylotypes were detected. Based on the sequencing results, a total of 4,059 OTUs were found in all of the feces samples. Compared with the initial microbial community on day 1 , the Sobs, Shannon diversity indices and Chao1 of the microbial communities in all three treatments on day 21 were decreased significantly $(p<0.05)$. The results indicated that neither EBS nor EBL had any effect on the microbiota diversity of the feces samples.

Relative read abundance of different bacterial families and genuses within different communities was found in the feces of different treatment groups. The bacteria whose relative abundance was $>1 \%$ at the family and genus levels for all treatment groups are shown in Fig. 1. The one-way ANOVA bar plots of the family and genus of the main fecal bacteria in weaned piglets are shown in Fig. 2. The relative abundance of Prevotellaceae, Acidaminococcaceae and Veillonellaceae in the feces of weaned piglets in the control

Table 4. Effects of probiotics on short-chain fatty acids in fecal samples of weaned piglets.

\begin{tabular}{lccc}
\hline Treatment & Control & EBS & EBL \\
\hline Acetate, $\mathrm{mM} / \mathrm{g}$ & $70.74 \pm 19.10^{\mathrm{a}}$ & $88.78 \pm 15.11^{\mathrm{b}}$ & $118.21 \pm 19.02^{\mathrm{c}}$ \\
Butyrate, $\mathrm{mM} / \mathrm{g}$ & $3.59 \pm 1.64^{\mathrm{a}}$ & $3.16 \pm 1.01^{\mathrm{a}}$ & $4.97 \pm 1.93^{\mathrm{b}}$ \\
Propionate, $\mathrm{mM} / \mathrm{g}$ & $29.65 \pm 9.88^{\mathrm{a}}$ & $34.18 \pm 9.94^{\mathrm{b}}$ & $48.46 \pm 9.66^{\mathrm{b}}$ \\
Valerate, $\mathrm{mM} / \mathrm{g}$ & $15.91 \pm 5.82^{\mathrm{a}}$ & $18.54 \pm 7.36^{\mathrm{a}}$ & $25.29 \pm 4.55^{\mathrm{b}}$ \\
\hline
\end{tabular}

${ }^{\mathrm{abc}}$ Means with different superscripts differ significantly $(p<0.05)$. 
Table 5. Effects of probiotics on the diversity of microbial communities in fecal samples of weaned piglets.

\begin{tabular}{lcccc}
\hline Items & day1_control & day21_control & day21_EBS & day21_EBL \\
\hline OTUs & $1172^{\mathrm{a}}$ & $1020^{\mathrm{a}}$ & $1005^{\mathrm{a}}$ & $1062^{\mathrm{a}}$ \\
Sobs & $599.44 \pm 64.35^{\mathrm{a}}$ & $474.92 \pm 76.39^{\mathrm{b}}$ & $401.07 \pm 120.34^{\mathrm{b}}$ & $417.25 \pm 124.1^{\mathrm{b}}$ \\
Shannon & $4.77 \pm 0.22^{\mathrm{a}}$ & $4.13 \pm 0.38^{\mathrm{b}}$ & $3.92 \pm 0.45^{\mathrm{b}}$ & $4.11 \pm 0.79^{\mathrm{b}}$ \\
Chao1 & $693.92 \pm 67.90^{\mathrm{a}}$ & $486.37 \pm 169.24^{\mathrm{b}}$ & $586.96 \pm 171.57^{\mathrm{b}}$ & $550.99 \pm 117.73^{\mathrm{b}}$ \\
\hline
\end{tabular}

${ }^{\mathrm{abc}}$ Means with different superscripts differ significantly $(p<0.05)$.

day1_control, the day before weaning; day21_control, basal diet supplemented with whey powder (no bacteria) on day 21 after weaning; day21_EBS, basal diet supplemented with EBS complex (Enterococcus faecium DSM 7134, Bacillus subtilis AS1.836 and Saccharomyces cerevisiae ATCC 28338) on day 21 after weaning; day21_EBL, basal diet supplemented with EBL complex (Enterococcus faecium DSM 7134, Bacillus subtilis AS1.836 and Lactobacillus paracasei L9) on day 21 after weaning.

treatment was significantly higher on day 21 than on day 1 $(p<0.001)$. The relative abundance of Lactobacillaceae in the control treatment was likewise significantly higher on day 21 than on day $1(p<0.05)$. However, the relative abundance of Ruminococcaceae, Bacteroidales S24-7, Christensenellaceae and Rikenellaceae in the feces of control treatment piglets on day 21 was significantly lower than on day $1(p<0.001)$. On day 21, piglets in the EBS treatment had a significantly higher relative abundance of Acidaminococcaceae $(p<0.01)$ and Streptococcaceae $(p<0.05)$ in their feces than piglets in the control treatment. Piglets in the EBL treatment had higher relative abundance of Erysipelotrichaceae $(p<0.05)$ and lower relative abundance of Bacteroidales S24-7 $(p<0.05)$ than piglets in control treatment.

The relative abundance of Prevotella_9 in the feces of weaned piglets in the control treatment $(p<0.001)$ and the relative abundance of Prevotella_1 and Lactobacillius on day 21 $(p<0.05)$ were also significantly higher than on day $1(p<0.05)$. However, the relative abundance of Clostridium_sensu_1 in the control treatment on day 21 was significantly lower than on day $1(p<0.01)$, while the relative abundance of Norank_f_Bacteroidales S24-7_group, Christensenellaceae_R7_group and Ruminococcaceae_UGG-005 in the feces of control treatment piglets on day 21 were significantly
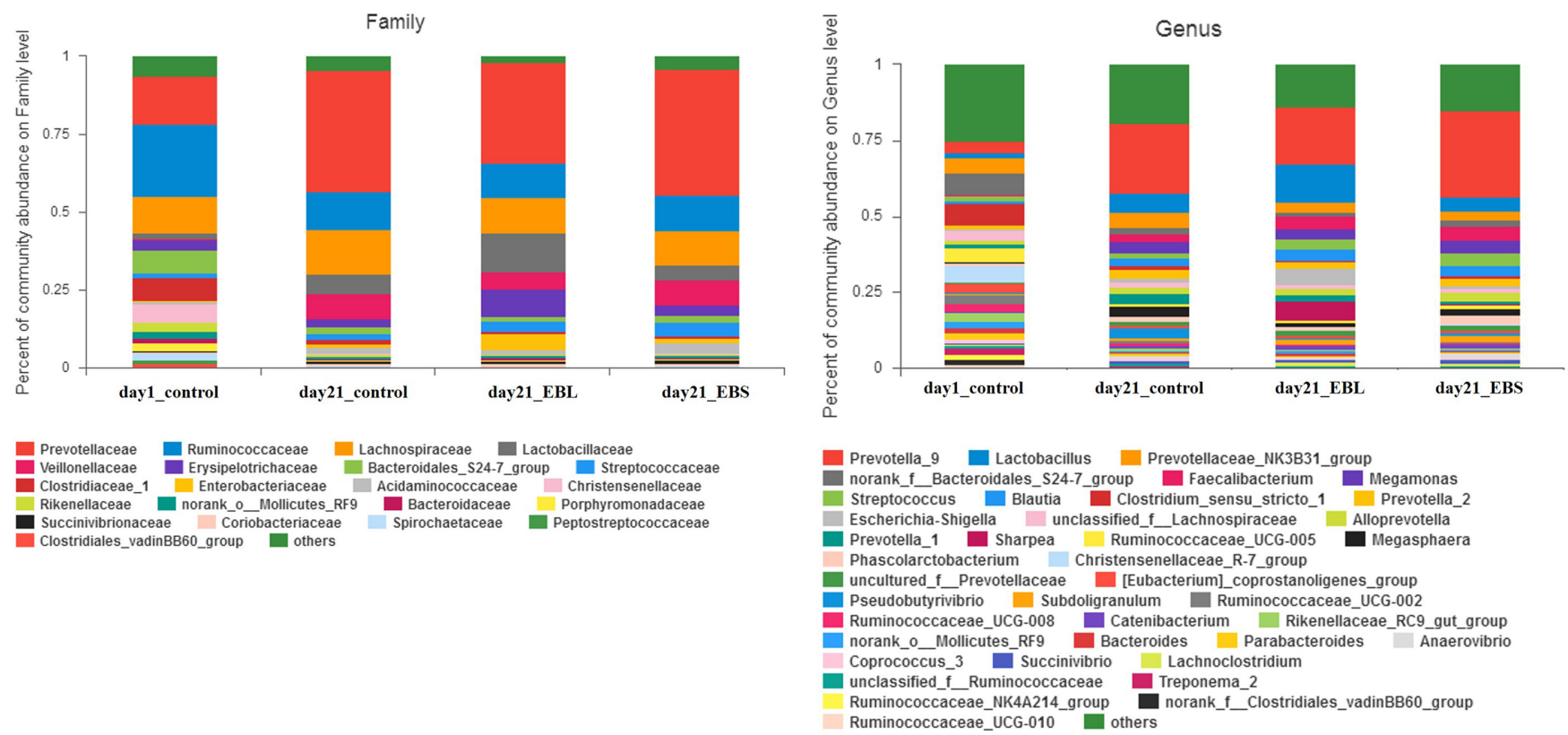

Fig. 1. Effects of probiotics on fecal bacterial communities on family and genus levels in the feces of weaned piglets.

Values are means; $n=16$. Families and genera with proportions less than $1 \%$ are not listed. day1_control, the day before weaning; day21_control, basal diet supplemented with whey powder (no bacteria) on day 21 after weaning; day21_EBS, basal diet supplemented with EBS complex (Enterococcus faecium DSM 7134, Bacillus subtilis AS1.836 and Saccharomyces cerevisiae ATCC 28338) on day 21 after weaning; day21_EBL, basal diet supplemented with EBL complex (Enterococcus faecium DSM 7134, Bacillus subtilis AS1.836 and Lactobacillus paracasei L9) on day 21 after weaning. 


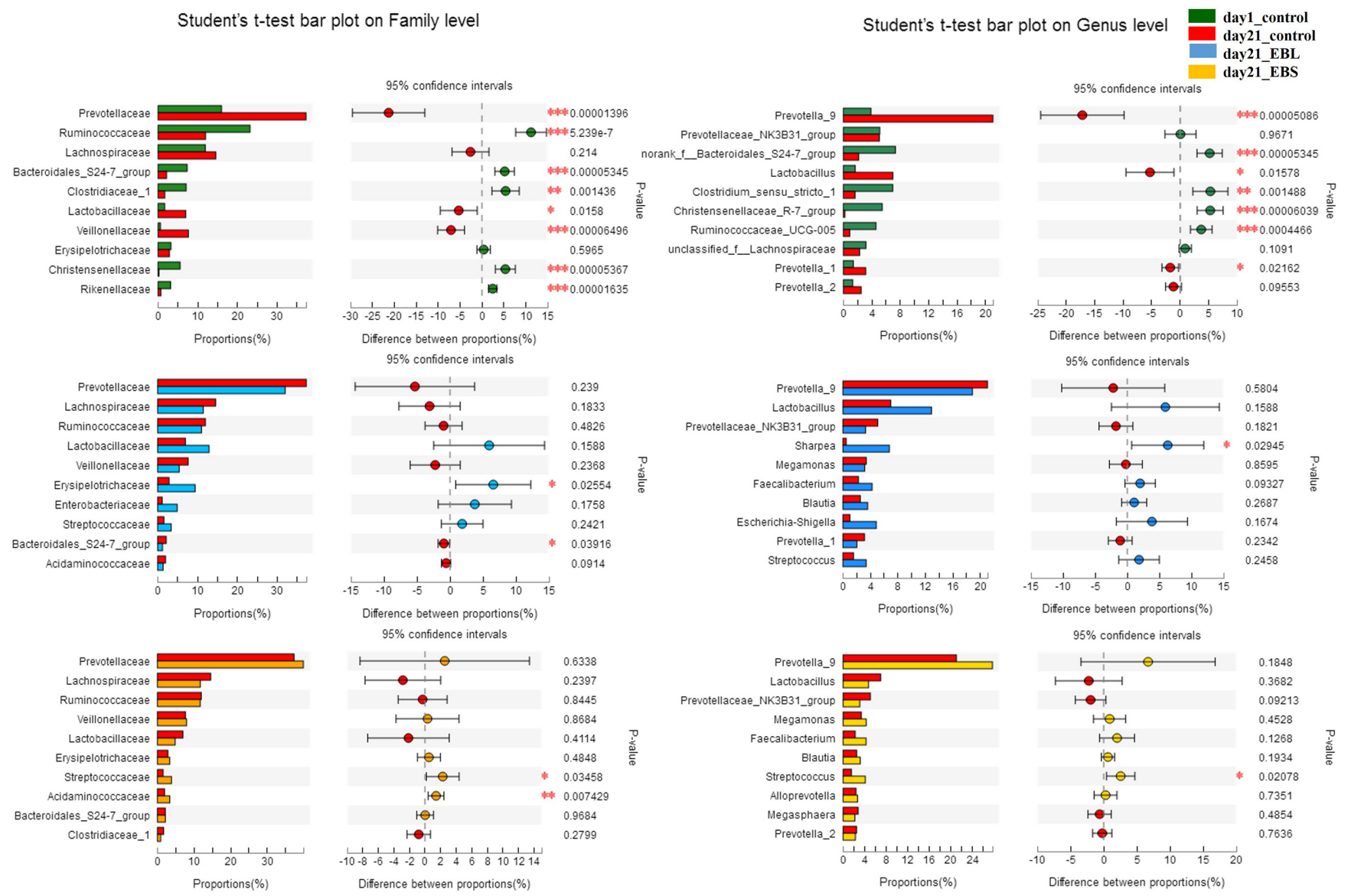

Fig. 2. One-way ANOVA bar plots showing predominant bacterial families and genuses in the feces of weaned piglets.

*Significant differences $(p<0.05),{ }^{* *}$ significant differences $(p<0.01)$, ${ }^{* * *}$ very significant differences $(p<0.001)$. day1_control, the day before weaning; day21_control, basal diet supplemented with whey powder (no bacteria) on day 21 after weaning; day21_EBS, basal diet supplemented with EBS complex (Enterococcus faecium DSM 7134, Bacillus subtilis AS1.836 and Saccharomyces cerevisiae ATCC 28338) on day 21 after weaning; day21_EBL, basal diet supplemented with EBL complex (Enterococcus faecium DSM 7134, Bacillus subtilis AS1.836 and Lactobacillus paracasei L9) on day 21 after weaning.

lower than on day $1(p<0.001)$. On day 21 , piglets in the EBL treatment had higher relative abundance of Sharpea than piglets in the control treatment $(p<0.05)$. And piglets in the EBS treatment had a significantly higher relative abundance of Streptococcus in their feces than piglets in the control treatment $(p<0.05)$.

According to the PCoA based on unweighted UniFrac distances presence discrepancy among samples, the microbial communities were clustered into the three groups by time and feed addition at at the genus level (Fig. 3). It is obvious that the fecal samples on day 1 and day 21 were separated (Fig. 3). The microbial communities of day21_EBS were clustered with day21_control, and those of day21_EBL treatment were clustered into different groups from them at the genus level.

\section{Discussion}

Weaning stress in piglets may cause changes in the gut environment and morphology that can result in low feed intake, high diarrhea incidence and imbalanced intestinal microbiota [17]. In the current study, supplementing the diet of post-weaning piglets with probiotic complexes increased ADG and improved ADFI and FCR compared with these values in control piglets fed an unsupplemented diet. These improvements could have resulted from the higher nutrient digestibility in EBS-fed and EBL-fed piglets. These findings are supported by earlier studies that showed positive effects on feed intake and growth performance in piglets fed diets supplemented with a complex of lactic acid bacteria during a 21-day post-weaning 


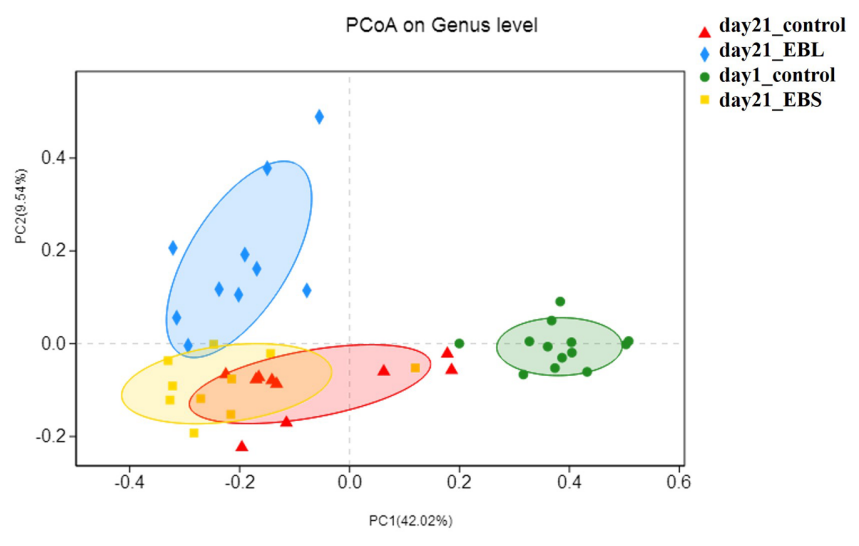

Fig. 3. PCoA of fecal bacterial communities at genus level. day1_control, the day before weaning; day21_control, basal diet supplemented with whey powder (no bacteria) on day 21 after weaning; day21_EBS, basal diet supplemented with EBS complex (Enterococcus faecium DSM 7134, Bacillus subtilis AS1.836 and Saccharomyces cerevisiae ATCC 28338) on day 21 after weaning; day21_EBL, basal diet supplemented with EBL complex (Enterococcus faecium DSM 7134, Bacillus subtilis AS1.836 and Lactobacillus paracasei L9) on day 21 after weaning.

period [18]. The enhanced growth performance observed in our study is consistent with previous published reports [9, $19,20]$.

Increased SCFAs in the piglets could help maintain normal gut function by performing a variety of regulatory functions in the host's metabolism [21], since butyric acid serves as an important energy source for gut epithelia and proliferation and differentiation of epithelial cells [22]. In the present study, levels of acetic acid and propionic acid of fecal samples in EBS and EBL treatments are significantly increased $(p<0.05)$. Besides, the EBL treatment also significantly increased butyrate content of fecal samples as compared with both control and EBS treatments. This result is in agreement with Mori $\mathrm{K}$ et al. [23], who reported that daily probiotic intake could increase the SCFAs levels in fecal samples of pigs. We could conclude that the reason the EBS and EBL treatments had more SCFAs observed is probably due to the probiotic complexes that improved intestinal microbial balance.

Consistent with the results of $\mathrm{Hu}$ et al. [6], the most dominant phyla of all fecal samples of weaned piglets were Firmicutes and Bacteroidetes, and higher bacterial diversity of piglets was observed in the initial microbial community on day 1 compared to all three treatments on day 21 ( $p<$ $0.05)$. Besides, the fecal microbiota obviously changed after weaning, which might be because of the changes of food intake and intestinal morphology of the piglets. At the family level, the relative abundance of Prevotellaceae, Lactobacillaceae and Veillonellaceae in feces of weaned piglets of the control treatment on day 21 were significantly enriched compared to day 1 in this study. However, the relative abundance of other families in feces of weaned piglets was lower. This result was in line with previous studies $[6,24,25]$. This is due to a dramatic change in microbial populations of pigs that usually happens when it comes to weaning or the withdrawal of milk [26]. Populations of saccharolytic microbes like Prevotellaceae and Lactobacillaceae increased mostly because the diet changed after weaning. The relative abundance of other families like opportunistic pathogens including Enterococcus in feces reduced, and this might be because their abundance usually decreases as the gut microbiota of weaned piglets matures.

In the present study, the relative abundance of Lactobacillaceae and Erysipelotrichaceae in feces of the EBL treatment group was higher than in the control treatment group on day 21. The elevated concentrations of SCFAs in faece of piglets in the EBL treatment could be the result of the higher numbers of Lactobacillus, and the major fermentation products of Lactobacillus like lactate produced by them that can be further metabolized to other SCFAs by bacteria in the gut [28]. It has been reported that a greater abundance of Sharpea within Erysipelotrichia which is a family of bacteria of the phylum Firmicutes may be related to diet and surroundings [29], while higher relative abundance of Erysipelotrichaceae may help mice gain weight faster [28]. As most members of the Firmicutes phylum degrade polysaccharide, the absorbing ability of weaned piglets in EBL treatment might be better than in the control treatment. On the contrary, the relative abundance of Bacteroidales within Bacteroidetes in the EBL treatment group is lower than in the control treatment group, while the relative abundance of the phylum Bacteroidetes was significantly elevated in diarrheic piglets [30]. Therefore, the lower abundance of Bacteroidetes may allow the piglets in EBL treatment to better adapt to weaning. These showed that dietary probiotics complexes additives could be efficient in decreasing PWD by adjusting the gut microbiota of piglets. Compared to day21_control, feeding of probiotics EBS could increase the relative abundance of Streptococcus, which is placed within the order Lactobacillales which usually increases SCFAs and might be beneficial to gut health [6]. The results revealed that improvement of the growth and nutrition absorbtion of weaned piglets compared to those in the control treatment group during the weaning period might be due to the higher concentrations of SCFAs 
in the EBS treatment group produced by beneficial bacteria.

The PCoA plot indicated that the microbial communities of day21_EBS were clustered with day21_control at genus level, for the reason that the community diversity microbes in feces of the EBS treatment group was similar with the control treatment group. Since Saccharomyces cerevisiae have little effect on the gut microbiota of piglets [27], they do not regulate the gut microbiota by colonization. The EBL with Lactobacillus paracasei L9 had a more favorable effect on fecal microbial communities than the use of EBS, and the day21_EBL treatment was clustered into different groups from the control treatment. This result is in the line with the findings that Lactobacilli significantly modify intestinal microbiota in piglets, and reduce the PWD of piglets [9].

Our results suggest that the complexes tested had probiotic properties in weaned piglets and regulated the composition of the intestinal microbiota. Moreover, supplementing the diet of weaned piglets with a probiotic complex improved ADG, ADFI and FCR, and reduced PWD after weaning for 21 days. The addition of a probiotic complex improved post-weaning performance, most likely by altering the bacterial community and some metabolites, such as SCFAs, in the feces of weaned piglets. In conclusion, the results of this study suggest that the addition of EBS and EBL may play an important role in the porcine digestive system and the prevention of diarrhea, especially during the weaning period.

\section{Acknowledgments}

The research was supported by the National Natural Science Foundation of China (31401668, 31601443) and the Beijing Science and Technology Project (Z181100009318005).

\section{Conflict of Interest}

The authors have no financial conflicts of interest to declare.

\section{References}

1. Nadeau É, Fairbrother JM, Zentek J, Bélanger L, Tremblay D, Tremblay CL, et al. 2017. Efficacy of a single oral dose of a live bivalent $E$. coli vaccine against post-weaning diarrhea due to F4 and F18-positive enterotoxigenic E. coli. Vet. J. 226: 32-39.

2. Jørgensen JN, Laguna JS, Millán C, Casabuena O, Gracia MI. 2016. Effects of a Bacillus -based probiotic and dietary energy content on the performance and nutrient digestibility of wean to finish pigs. Anim. Feed Sci. Technol. 221: 54-61.

3. Dou S, Gadonna-Widehem P, Rome V, Hamoudi D, Rhazi L, Lakhal L, et al. 2017. Characterisation of early-life fecal microbiota in susceptible and healthy pigs to post-weaning diarrhoea. PLoS One 12: e0169851.

4. Xu YG, Yu H, Zhang L, Liu M, Qiao XY, Cui W, et al. 2016. Probiotic properties of genetically engineered Lactobacillus plantarum producing porcine lactoferrin used as feed additive for piglets. Process Biochem. 51: 719-724.

5. Rhouma M, Fairbrother JM, Beaudry F, Letellier A. 2017. Post weaning diarrhea in pigs: risk factors and non-colistinbased control strategies. Acta Vet. Scand. 59: 31.

6. Hu J, Nie Y, Chen J, Zhang Y, Wang Z, Fan Q, et al. 2016. Gradual changes of gut microbiota in weaned miniature piglets. Front. Microbiol. 7: 1727.

7. García KE, de Souza TCR, Landín GM, Barreyro AA, Santos MGB, Soto JGG. 2014. Microbial fermentation patterns, diarrhea incidence, and performance in weaned piglets fed a low protein diet supplemented with probiotics. Food Nutr. Sci. 05: 1776-1786.

8. Hou C, Zeng X, Yang F, Liu H, Qiao S. 2015. Study and use of the probiotic Lactobacillus reuteri in pigs: a review. J. Anim. Sci. Biotechnol. 6: 14.

9. Su Y, Chen X, Liu M, Guo X. 2017. Effect of three lactobacilli with strain-specific activities on the growth performance, faecal microbiota and ileum mucosa proteomics of piglets. J. Anim. Sci. Biotechnol. 8: 52.

10. Giang HH, Viet TQ, Ogle B, Lindberg JE. 2010. Growth performance, digestibility, gut environment and health status in weaned piglets fed a diet supplemented with potentially probiotic complexes of lactic acid bacteria. Livest Sci. 129: 95-103.

11. Liu X, Wang Y, Wang H, Lu W. 2014. Effect of a liquid culture of Enterococcus faecalis CGMCC1.101 cultivated by a high density process on the performance of weaned piglets. Livest Sci. 170: 100-107.

12. Prieto ML, O'Sullivan L, Tan SP, McLoughlin P, Hughes $H$, O'Donovan O, et al. 2014. Evaluation of the efficacy and safety of a marine-derived Bacillus strain for use as an infeed probiotic for newly weaned pigs. PLoS One 9: e88599.

13. Yang J, Ren F, Zhang H, Jiang L, Hao Y, Luo X. 2015. Induction of regulatory dendritic cells by Lactobacillus paracasei L9 prevents allergic sensitization to bovine $\beta$-lactoglobulin in mice. J. Microbiol. Biotechnol. 25: 1687-1696.

14. Pierce KM, Callan JJ, McCarthy P, O’Doherty JV. 2007. Performance of weanling pigs offered low or high lactose diets supplemented with avilamycin or inulin. Anim. Sci. 80: 313-318.

15. Yang KM, Jiang ZY, Zheng CT, Wang L, Yang XF. 2014. Effect of Lactobacillus plantarum on diarrhea and intestinal barrier function of young piglets challenged with enterotoxigenic Escherichia coli K88. J. Animal Sci. 92: 1496-1503.

16 Geng MM, Li-Wei XU, Yuan HZ, Wang JR, Kong XF, Wang M. 
2015. A determination method based on gas chromatography for analysis of short-chain fatty acids in colonic contents of piglet. Progress Modern Biomed. 15: 1010-1014.

17. Hu Y, Dun Y, Li S, Zhao S, Peng N, Liang Y. 2014. Effects of Bacillus subtilis $\mathrm{KN}-42$ on growth performance, diarrhea and faecal bacterial flora of weaned piglets. Asian-Australas J. Anim. Sci. 27: 1131-1140.

18. Giang HH, Viet TQ, Ogle B, Lindberg JE. 2012. Growth performance, digestibility, gut environment and health status in weaned piglets fed a diet supplemented with a complex of lactic acid bacteria alone or in combination with Bacillus subtilis and Saccharomyces boulardii. Livest Sci. 143: 132-141.

19. Dong X, Zhang N, Zhou M, Tu Y, Deng K, Diao Q. 2014. Effects of dietary probiotics on growth performance, faecal microbiota and serum profiles in weaned piglets. Animal Production Sci. 54: 616-621.

20. Liu C, Zhu Q, Chang J, Yin Q, Song A, Li Z, et al. 2017. Effects of Lactobacillus casei and Enterococcus faecalis on growth performance, immune function and gut microbiota of suckling piglets. Arch. Anim. Nutr. 71: 120-133.

21. Den BG, Van EK, Groen AK, Venema K, Reijngoud DJ, Bakker BM. 2013. The role of short-chain fatty acids in the interplay between diet, gut microbiota, and host energy metabolism. J. Lipid Res. 54: 2325-2340.

22. Sakata T, Adachi M, Hashida M, Sato N, Kojima T. 1995. Effect of n-butyric acid on epithelial cell proliferation of pig colonic mucosa in short-term culture. Dtsch Tierarztl Wochenschr. 102: 163-164.

23. Mori K, Ito T, Miyamoto H, Ozawa M, Wada S, Kumagai Y, et al. 2011. Oral administration of multispecies microbial supplements to sows influences the composition of gut microbiota and fecal organic acids in their post-weaned piglets. J. Biosci. Bioeng. 112: 145-150.

24. Gresse R, Chaucheyras-Durand F, Fleury MA, Van de Wiele T, Forano E, Blanquet-Diot S. 2017. Gut microbiota dysbiosis in postweaning piglets: understanding the keys to health. Trends Microbiol. 25: 851-873

25. Chen WB, Cheng MJ, Tian YB, Wang QH, Wang B, Li MJ, et al. 2017. Effects of Armillariella tabescens mycelia on the growth performance and intestinal immune response and microflora of early-weaned pigs. Anim. Sci. J. 88: 1388-1397.

26. Frese SA, Parker K, Calvert CC, Mills DA. 2015. Diet shapes the gut microbiome of pigs during nursing and weaning. Microbiome 3: 28.

27. Czech A, Smolczyk A, Ognik K, Wlazło Ł, NowakowiczDębek B, Kiesz M. 2018. Effect of dietary supplementation with yarrowia lipolytica or saccharomyces cerevisiae yeast and probiotic additives on haematological parameters and the gut microbiota in piglets. Res. Vet. Sci. 119: 221-227.

28. Ushida K, Hoshi S. 2009. 13 C-NMR studies on lactate metabolism in a porcine gut microbial ecosystem. Microb. Ecol. Health Dis. 14: 242-247.

29. Greiner T, Bäckhed F. 2011. Effects of the gut microbiota on obesity and glucose homeostasis. Trends Endocrinol Metab. 22: $117-123$

30. Yang Q, Huang X, Zhao S, Sun W, Yan Z, Wang P, et al. 2017. Structure and function of the fecal microbiota in diarrheic neonatal piglets. Front Microbiol. 8: 502. 